\title{
AVALIAÇÃO DO ARMAZENAMENTO TÉRMICO NA GERAÇÃOO DE POTÊNCIA EM CENTRAIS TERMOSSOLARES
}

\section{EVALUATION OF THERMAL STORAGE IN THE GENERATION OF POWER IN THERMOSOLAR CENTERS}

\author{
P. H. F. GREPINO ${ }^{1 *}$, A. M. O. SIQUEIRA ${ }^{1}$
}

${ }^{1}$ Universidade Federal de Viçosa, Departamento de Química, Viçosa, Minas Gerais, Brasil

${ }^{*}$ Universidade Federal de Viçosa, Departamento de Química, Viçosa, Minas Gerais, Brasil: +55 (31) 9 8646-9714

e-mail: paulo.grepino@gmail.com (P.H.F.GREPINO).

\section{A R T I C L E I N F O}

\section{Article history:}

Received 2017-11-26

Accepted 2018-04-05

Available online 2018-06-30

\section{palavras-chave \\ Energia Solar \\ Armazenamento Térmico \\ Simulação Numérica}

\section{keywords}

Solar Energy

Thermal Storage

Numerical Simulation

\begin{abstract}
A B S T R A C T
Taking into account the environmental impacts generated by fossil energy sources, their limitations regarding availability and exhaustion in the near future, this work seeks to evaluate, through the numerical simulation performed in the TRNSYS software, the technical feasibility of storing solar thermal energy for its use in power generation in solar thermal power plants. The use of such technology implied a considerable increase of hours in which power was generated when compared to the system without this resource, increased from 3241 to 8583 hours of power generated in a period of one year, which corresponds to an increase of $260 \%$. Factors such as the insufficient number of studies in the area and the high economic impact for its installation and operation still limit the competitiveness of such technology in relation to other models already established in the market. However, this presented technical potential to fill the gaps caused by the variability of the solar source.
\end{abstract}

R E S U M O

Levando em consideração os impactos ambientais gerados pelas fontes fósseis de energia, suas limitações quanto à disponibilidade e esgotamento em um futuro próximo, este trabalho busca avaliar, através da simulação numérica realizada no software TRNSYS, a viabilidade técnica do armazenamento de energia térmica de fonte solar para sua utilização na geração de potência em centrais termossolares. A utilização de tal tecnologia implicou no aumento considerável de horas em que houve geração de potência quando comparado ao sistema sem este recurso, aumentado de 3241 para 8583 horas de potência gerada no período de um ano, o que corresponde a um aumento de $260 \%$. Fatores como a quantidade insuficiente de estudos na área e o alto impacto econômico para sua instalação e operação ainda limitam a competitividade de tal tecnologia diante de outros modelos já estabelecidos no mercado. Porém, essa apresentou potencial técnico para preencher as lacunas oriundas da variabilidade da fonte solar. 


\section{N O M E N C L A T U R A}

\begin{tabular}{|c|c|}
\hline$C p_{\text {água }}$ & Capacidade calorífica da água $\left[\mathrm{kJ} \cdot \mathrm{kg}^{-1} \cdot{ }^{\circ} \mathrm{C}^{-1}\right]$ \\
\hline$C p_{\text {material }}$ & 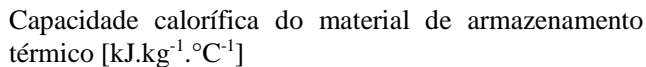 \\
\hline$C p_{\text {vapor }}$ & Capacidade calorífica do vapor d'água $\left[\mathrm{kJ} . \mathrm{kg}^{-1} \cdot{ }^{\circ} \mathrm{C}^{-1}\right]$ \\
\hline$h_{1}$ & Entalpia específica do estado termodinâmico $1\left[\mathrm{~kJ}^{\mathrm{kg}} \mathrm{kg}^{-1}\right]$ \\
\hline$h_{2}$ & Entalpia específica do estado termodinâmico $2\left[\mathrm{~kJ} \cdot \mathrm{kg}^{-1}\right]$ \\
\hline$h_{3}$ & Entalpia específica do estado termodinâmico $3\left[\mathrm{~kJ}^{2} \mathrm{~kg}^{-1}\right]$ \\
\hline$h_{4}$ & Entalpia específica do estado termodinâmico $4\left[\mathrm{~kJ} \cdot \mathrm{kg}^{-1}\right]$ \\
\hline$h_{5}$ & Entalpia específica do estado termodinâmico $5\left[\mathrm{~kJ} \mathrm{~kg}^{-1}\right]$ \\
\hline$h_{6}$ & Entalpia específica do estado termodinâmico $6\left[\mathrm{~kJ}^{\mathrm{kg}} \mathrm{kg}^{-1}\right]$ \\
\hline$h_{7}$ & Entalpia específica do estado termodinâmico $7\left[\mathrm{~kJ}^{\mathrm{kg}} \mathrm{kg}^{-1}\right]$ \\
\hline$h_{l s}$ & Entalpia específica do líquido saturado $\left[\mathrm{kJ}^{\mathrm{k}} \mathrm{kg}^{-1}\right]$ \\
\hline$h_{v s}$ & Entalpia específica do vapor saturado $\left[\mathrm{kJ}^{2} \mathrm{~kg}^{-1}\right]$ \\
\hline$y$ & Fração de vapor extraída na turbina de alta pressão [ ] \\
\hline$\dot{m}$ & Vazão mássica de fluido de trabalho $\left[\mathrm{kg} \cdot \mathrm{s}^{-1}\right]$ \\
\hline$\dot{m}_{\text {água }}$ & Vazão mássica de água $\left[\mathrm{kg} . \mathrm{s}^{-1}\right]$ \\
\hline$\dot{m}_{\text {vapor }}$ & Vazão mássica de água [kg.s $\left.{ }^{-1}\right]$ \\
\hline$m_{\text {material }}$ & Massa de material para o armazenamento térmico $[\mathrm{kg}]$ \\
\hline$\eta_{c}$ & Eficiência do Ciclo de Rankine [ ] \\
\hline$\dot{Q}$ & Potência demandada no Ciclo de Rankine $[\mathrm{kW}]$ \\
\hline$\dot{Q}_{\text {noite }}$ & Potência noturna demandada no Ciclo de Rankine [kW] \\
\hline$Q_{\text {armazenado }}$ & Energia noturna demandada no Ciclo de Rankine $[\mathrm{kJ}]$ \\
\hline$\dot{W}_{b}$ & Trabalho adicionado às bombas $[\mathrm{kW}]$ \\
\hline$\dot{W}_{c}$ & $\begin{array}{l}\text { Energia gerada por unidade de massa de fluido de } \\
\text { trabalho }\left[\mathrm{kJ} \mathrm{kg}^{-1}\right]\end{array}$ \\
\hline$\dot{W}_{t}$ & Trabalho extraído nas turbinas $[\mathrm{kW}]$ \\
\hline
\end{tabular}

\section{INTRODUÇÃO}

De acordo com o relatório do ano de 2016 disponibilizado pelo REN21 - Renewable Energy Policy Network for the 21st Century - os combustíveis fósseis foram responsáveis, até 2015 , por $76,3 \%$ da energia primária mundial. Combustíveis esses que vêm sendo associadas aos impactos ambientais registrados nas últimas décadas, o que leva a pensar na necessidade da busca por outras vertentes energéticas que não contribuam para tais efeitos. Além disso, deve-se levar em consideração o fato de que tais fontes são limitadas e que o uso contínuo das mesmas implicará em seus esgotamentos e, por conseguinte, aumento gradativo de seus preços.

Por serem energias limpas cujas fontes são inesgotáveis, a utilização de energias renováveis é cada vez mais estudada, incentivada e otimizada (Costa, 2014). Segundo McGrath (2016), a utilização de fontes alternativas de energia cresceu nos últimos anos e vem ganhando destaque, principalmente, em países emergentes. Um total de aproximadamente $147 \mathrm{GW}$ de capacidade foram instalados em vários países do mundo até 2015 e, de acordo com a Agência Internacional de Energia, a capacidade global instalada deve subir em $40 \%$ até 2020 (Outlook, 2016).

Segundo Ter-Gazarian (2011) a radiação solar que atinge a Terra é da ordem de 170 mil TW, valor que supera em grande quantidade os fluxos de outras fontes energéticas como ventos e marés, por exemplo. Dessa forma, devido sua alta disponibilidade, a energia solar tem sido considerada uma fonte energética com grande potencial para exploração.

A fonte solar representou cerca de $1 \%$ do total da energia elétrica produzida no mundo em 2014 (SolarPower Europe, 2014). No entanto, o uso das tecnologias para geração elétrica que utilizam o sol como fonte tem crescido substancialmente nos últimos anos, especialmente a fotovoltaica, que passou de 2,6 GWp para 177 GWp, entre 2004 e 2014 - crescimento anual de 52,5\% (Sawin; Sverrisson; Ren21, 2015). O aumento na capacidade instalada também se traduziu em um aumento nos investimentos. Em 2014, estima-se que tenham sido investidos cerca de 150 bilhões de dólares em energia solar, 55\% do total investido em energias renováveis no mesmo ano (McCrone et al., 2015).

A produção de eletricidade através da energia solar é possível através de células fotovoltaicas ou pelo aquecimento de um fluido. No primeiro caso, as células são constituídas por sílica, fósforo e boro que, ao receberem os raios solares, originam a produção de eletricidade, que pode ser armazenada em baterias ou injetada diretamente na rede elétrica através de um inversor. No segundo caso, espelhos concentram a luz solar para vaporizar um fluido, que movimenta as pás de uma turbina a vapor produzindo eletricidade (Pinto, 2015). Tecnologias que se fazem da utilização de espelhos para concentração dos raios solares para geração de potência são chamadas Plantas Heliotérmicas ou Plantas de Concentração Solar - em inglês Concentrating Solar Power (CSP) - (IRENA, 2012).

Contudo, o aumento da composição de energias renováveis na matriz mundial se associa à necessidade do armazenamento dessas, devido à instabilidade e sazonalidade de tais fontes (Costa, 2014). Em outras palavras, pode-se dizer que, na maioria das vezes, a disponibilidade de tais fontes não corresponde com sua demanda. Para tanto, os sistemas de armazenamento de energia - em inglês Energy Storage System (ESS) - têm a capacidade de suprir essa lacuna, possibilitando a satisfação da requisição energética da sociedade bem como a solução de problemas de picos de energia, estabilidade e perturbações que afetam a qualidade energética, além de promover a redução do consumo, dos níveis de poluição e de custos energéticos (Cotterman, 2013; Dincer e Rosen, 2011; Huggins, 2010).

Em termos termodinâmicos, armazenamento de energia inclui armazenamento de calor transferido, o qual é contido antes de ser disponibilizado para fins úteis. Novos dispositivos de armazenamento avançados são muitas vezes parte integrante de outras novas tecnologias e, por vezes, estas podem ser tornadas mais viáveis por inovações nos mecanismos que compreendem este processo. Existem diversos métodos aplicados para o armazenamento energético, porém, a maioria deles ainda se encontra em processo de estudo. Os avanços no armazenamento beneficiam, especialmente, as tecnologias de energia eólica e solar (Dincer e Rosen, 2011).

Entre os métodos de armazenamento energético, pode-se citar o uso de materiais de mudança de fase - em inglês Phase Change Materials (PCMs) - aplicados no armazenamento de energia térmica na forma de calor latente. Os materiais de mudança de fase são substâncias com a capacidade de armazenar calor na forma de calor latente e fornecer uma densidade de energia térmica elevada durante o processo de mudança de fase (Tristão, 2014). Também, há o armazenamento de calor sensível, o que é possível devido a variação de temperatura do suporte de armazenamento. A energia armazenada pode ser utilizada, quando necessário, para vaporização de um fluido, o qual será direcionado para geração de energia elétrica em turbinas.

Como toda tecnologia em fase de introdução no mercado, a heliotermia tem de enfrentar alguns desafios. Apesar do constante desenvolvimento, a tecnologia ainda não chegou a um grau de maturidade necessário para competir diretamente com as fontes de energia já estabelecidas no mercado. Tendo em vista 
estes fatores, este trabalho propõe avaliar a viabilidade técnica do armazenamento térmico de calor sensível, proveniente da energia solar, para fins de geração de potência na ordem de 100 MW em centrais termossolares o que representa, em média, o consumo de uma cidade de 400 mil habitantes. A avaliação foi realizada pelo procedimento de simulação numérica, desenvolvida no software TRNSYS, tomando como base dados meteorológicos do município de Recife-PE, disponíveis na biblioteca do software utilizado. A escolha da cidade foi fundamentada em sua maior disponibilidade de Irradiação Normal Direta - em inglês Direct Normal Irradiation (DNI) comparada à outras localidades do Brasil, na ordem de 6,26 GJ.m ${ }^{-2} \cdot$ ano $^{-1}$.

\section{REVISÃO DA LITERATURA}

\subsection{Armazenamento Térmico}

Um sistema de armazenamento térmico - em inglês Thermal Energy Storage (TES) - é composto basicamente de três itens principais: o material de armazenamento, o equipamento de transferência de calor e o reservatório térmico. Quanto ao material de armazenamento, deve-se levar em consideração a faixa de temperatura de operação do acumulador e suas propriedades termofísicas. $\mathrm{O}$ equipamento de transferência de calor tem como função o transporte de energia da fonte quente para o fluido de trabalho, na operação de carregamento, e do fluido para o consumo, na operação de descarregamento. Podese efetuar essa transferência de calor por intermédio de trocadores de calor (modo indireto) ou usando o próprio fluido de trabalho (modo direto). O reservatório térmico tem a função de confinar o fluido de trabalho e separá-lo do meio ambiente, limitando de maneira efetiva as perdas de calor pelos contornos físicos (Pantuzi, 2006).

Um critério que torna o armazenamento térmico mais vantajoso quando comparado ao armazenamento de outras energias renováveis como a eólica e a solar fotovoltaica é a possibilidade de armazenar o calor por métodos relativamente baratos (Wendel, 2010). Há aplicações e, ainda, estão em desenvolvimento diversas concepções de armazenamento térmico adequados para utilização em Plantas Heliotérmicas.

Armazenamento térmico por calor sensível: Neste tipo de sistema a energia é acumulada devido à mudança de temperatura do suporte de armazenamento. A capacidade de armazenar calor sensível depende em grande medida do valor do calor específico volumétrico. Este descreve a capacidade de um determinado volume de uma substância armazenar energia enquanto sofre alteração de temperatura, sem mudança de fase. Para ser atrativo para aplicações TES um material deve ser barato, ter um calor específico elevado e ter uma alta taxa de libertação e de extração de calor, ou seja, alta capacidade calorífica e alta condutividade térmica (Dincer e Rosen, 2011). A Tabela 1 apresenta algumas propriedades termofísicas e as temperaturas limites de operação de alguns materiais comercialmente utilizados em sistemas para tal finalidade.

Tabela 1 - Propriedades termofísicas de materiais aplicados em sistemas TES. (Adaptado de Kuravi et. al (2013))

\begin{tabular}{|c|c|c|c|c|c|c|c|}
\hline $\begin{array}{l}T_{\text {frio }} \\
\left({ }^{\circ} \mathbf{C}\right)\end{array}$ & $\begin{array}{c}\mathbf{T}_{\text {quente }} \\
\left({ }^{\circ} \mathbf{C}\right)\end{array}$ & Material & $\begin{array}{c}\text { Condutividade } \\
\text { térmica } \\
\left(\mathbf{W} \cdot \mathbf{m}^{-1} \cdot \mathbf{K}^{-1}\right)\end{array}$ & $\begin{array}{c}\text { Massa } \\
\text { específica } \\
\left(\mathbf{k g} \cdot \mathbf{m}^{-3}\right)\end{array}$ & $\begin{array}{c}\text { Calor } \\
\text { specífico a pressã } \\
\text { constante } \\
\left(\mathbf{k J}^{-1} \mathbf{k g}^{-1} \cdot \mathbf{K}^{-1}\right)\end{array}$ & $\begin{array}{c}\text { Capacidade } \\
\text { Térmica } \\
\text { volumétrica } \\
\left(\mathbf{k W h} \cdot \mathbf{m}^{-3}\right)\end{array}$ & $\begin{array}{c}\text { Estado } \\
\text { físico }\end{array}$ \\
\hline 200 & 300 & Areia-Rocha-Óleo & 1,0 & 1.700 & 1,30 & 60 & Sólido \\
\hline 200 & 400 & Concreto reforçado & 1,5 & 2.200 & 0,85 & 100 & Sólido \\
\hline 200 & 400 & Ferro fundido & 37,0 & 7.200 & 0,56 & 160 & Sólido \\
\hline 200 & 500 & $\mathrm{NaCl}$ & 7,0 & 2.160 & 0,85 & 150 & Sólido \\
\hline 200 & 700 & Aço fundido & 40,0 & 7.800 & 0,60 & 450 & Sólido \\
\hline 200 & 700 & Tijolo refratário (Sílica) & 1,5 & 1.820 & 1,00 & 150 & Sólido \\
\hline 200 & 1.200 & Tijolo refratário (Magnésia) & 5,0 & 3.000 & 1,15 & 600 & Sólido \\
\hline
\end{tabular}

Armazenamento térmico por calor latente: Nesta tecnologia, o processo de armazenamento térmico ocorre devido a mudança de fase do material de armazenamento. Dentre as mudanças de fase existentes com libertação ou absorção de calor, as mais apropriadas para os sistemas de armazenamento de energia térmica são as sólido-líquido, líquido-gás e sólidosólido. Em geral, os PCMs sólido-liquido são os mais utilizados, pois possuem baixa variação volumétrica ao mudarem de fase resultando em economia na construção do armazenamento (Costa, 2014). Entretanto, uma desvantagem desse procedimento em relação ao armazenamento de calor sensível é o fato de, devido a frequência que as mudanças de fase ocorrem, há maior desgaste do material o que implica em redução de sua vida útil. Para serem utilizados em aplicações reais, os materiais de acumulação de um sistema de armazenamento de calor latente devem obedecer a critérios térmicos, físicos, cinéticos, químicos e econômicos (Costa, 2014).

\section{MODELAGEM MATEMÁTICA E IMPLEMENTAÇÃO DO MODELO NO TRNSYS}

\subsection{Ciclo de Rankine}

O Ciclo de Rankine é um ciclo termodinâmico de geração de potência a partir de vapor d'água, o qual se baseia em quatro processos, sendo eles o bombeamento adiabático nas bombas, geração de vapor, expansão adiabática nas turbinas e condensação do vapor a pressão constante. Neste ciclo, o Fluido de Transferência de Calor - em inglês Heat Transfer Fluid $(H T F)$ - fornece energia para o aquecimento da água no préaquecedor, geração de vapor no gerador de vapor e superaquecimento do vapor até $390{ }^{\circ} \mathrm{C}$ e pressão de 100 bar. $\mathrm{O}$ vapor é, então, disponibilizado para movimentação das pás das turbinas, gerando, assim, potência útil. Adota-se, neste trabalho, um modelo adaptado do Ciclo de Rankine chamado de Ciclo de Rankine Regenerativo (Figura 1), modificado para aumentar a eficiência do ciclo em relação ao modelo original desenvolvido 
pelo Engenheiro Escocês William Rankine no século XIX.

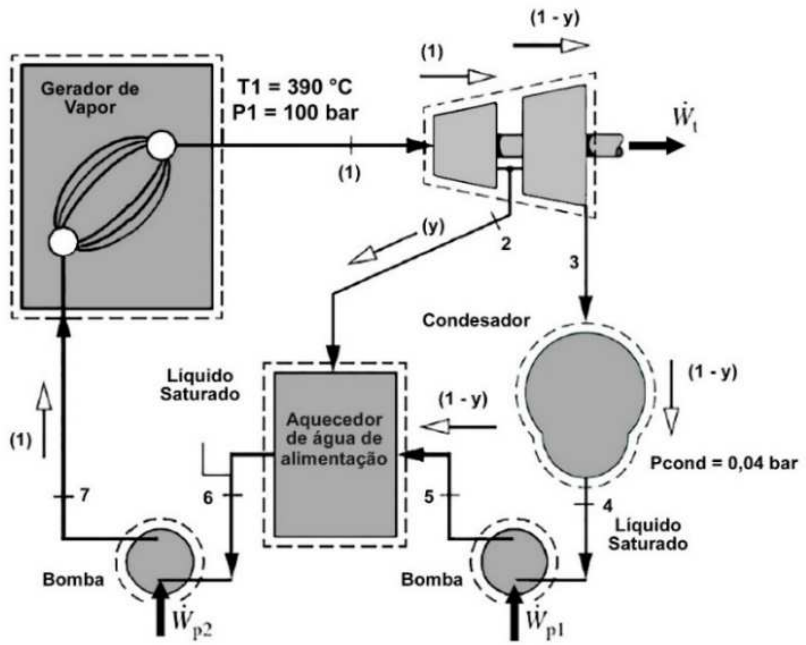

Figura 1 - Ciclo de Rankine Regenerativo. Adaptado de Moran et. al (2014)

Diagrama Temperatura-Entropia (T-S): Nesta seção é apresentado o diagrama $\mathrm{T}-\mathrm{S}$ que representa o ciclo termodinâmico de Rankine Regenerativo, tratado daqui pra frente apenas como Ciclo de Rankine, para efeito de simplificação, proposto para a geração de potência (Figura 2).

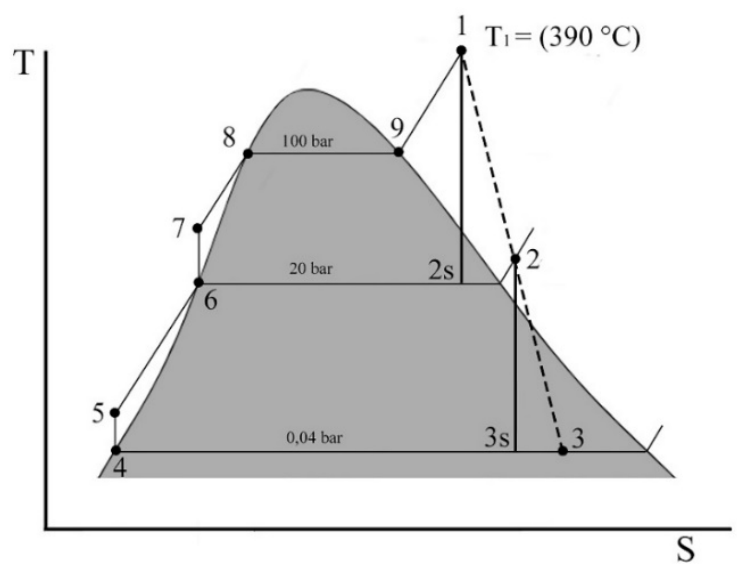

Figura 2 - Diagrama Temperatura-Entropia do ciclo de potência de Rankine. Adaptado de Strigari (2009)

A Tabela 2 apresenta os valores das entalpias específicas calculadas para os pontos do Ciclo de Rankine, destacados no diagrama T-S apresentado na Figura 2, adotando as seguintes considerações:

- Cada componente do ciclo é analisado como um volume de controle fixo.

- Todos os processos do fluido de trabalho são internamente reversíveis, exceto para as expansões através dos dois estágios da turbina e mistura no aquecedor de água de alimentação aberta.

- As turbinas, as bombas e o aquecedor de água de alimentação operam adiabaticamente.

- Os efeitos de energia cinética e potencial são desprezíveis.

- O fluido na saída do pré-aquecedor e do condensador se encontram como líquido saturado $(\mathrm{x}=0)$ e na saída do evaporador como vapor saturado $(\mathrm{x}=1)$.
Tabela 2 - Valores de entalpia específica. Estado Termodinâmico Entalpia Específica (kJ/kg)

$\begin{array}{cc}1 & 3.065,86 \\ 2 \mathrm{~s} & 2.714,70 \\ 2 & 2.767,37 \\ 3 \mathrm{~s} & 1.888,00 \\ 3 & 2.019,90 \\ 4 & 121,40 \\ 5 & 123,40 \\ 6 & 908,62 \\ 7 & 918,03 \\ 8 & 1.407,87 \\ 9 & 2.725,47\end{array}$

Extração de trabalho e adição de trabalho e calor: A produção total da turbina é calculada baseada em uma unidade de massa de vapor que passa pela mesma. Aplicando um balanço de massa e energia pode-se determinar a fração (y) de vapor extraída no estado 2 , ou seja, na turbina de alta pressão.

$$
y=\frac{h_{6}-h_{5}}{h_{2}-h_{5}}=29 \%
$$

Turbinas:

$$
\frac{\dot{w}_{t}}{\dot{m}}=\left(h_{1}-h_{2}\right)+(1-y)\left(h_{2}-h_{3}\right)=823,96 \frac{\mathrm{kJ}}{\mathrm{kg}}
$$

Bombas:

$$
\frac{\dot{W}_{b}}{\dot{m}}=\left(h_{7}-h_{6}\right)+(1-y)\left(h_{5}-h_{4}\right)=10,82 \frac{k J}{k g}
$$

Evaporador:

$$
\frac{\dot{Q}}{\dot{m}}=\left(h_{1}-h_{7}\right)=2.147,83 \frac{k J}{k g}
$$

Eficiência do ciclo: A eficiência térmica do ciclo é dada por:

$$
\eta_{c}=\frac{\dot{W}_{t}-\dot{W}_{b}}{\dot{Q}}=37,9 \%
$$

Vazão mássica de vapor demanda: A vazão mássica de vapor que alimenta o ciclo é determinada com base na potência desejada, $100 \mathrm{MW}$, conforme a seguinte relação:

$$
\begin{aligned}
& \dot{W}_{c}=\frac{\dot{W}_{t}}{\dot{m}}-\frac{\dot{W}_{b}}{\dot{m}}=813,14 \frac{\mathrm{kJ}}{\mathrm{kg}} \\
& \dot{m}=\frac{W_{\text {demandada }}}{\dot{W}_{c}}=\frac{100 \mathrm{MW}}{813,14 \frac{\mathrm{kJ}}{\mathrm{kg}}}=123 \frac{\mathrm{kg}}{\mathrm{s}}
\end{aligned}
$$

\subsection{Ciclo de Rankine Acoplado ao Campo Solar}

Realizada a modelagem do Ciclo de Rankine, pode-se acoplar a este o campo solar, o qual será responsável por captar a energia proveniente do Sol e direcioná-la para o aquecimento do Fluido de Transferência de Calor que conterá tal energia até sua utilização para geração de vapor à temperatura útil para a geração de potência. De acordo com os dados meteorológicos Meteonorm, disponíveis na biblioteca Weather do TRNSYS, para o município de Recife - PE, a energia solar por unidade de superfície em um ano de 8.760 horas é da ordem de $6,26 \mathrm{GJ} \mathrm{m}^{-2}$. Dessa forma, a potência solar média (PSM) pode ser calculada da seguinte maneira: 


$$
P S M=\frac{6,26 \frac{G J}{m^{2} a n o}}{8760 \mathrm{~h}}=0,199 \frac{\mathrm{kW}}{\mathrm{m}^{2}}
$$

A potência demandada, corrigida de acordo com a eficiência do ciclo, calculada na equação (5), é dividida pela potência solar média para calcular a área do campo solar:

$$
\text { Área do Campo Solar }=\frac{\frac{100}{0,379} \mathrm{MW}}{0,199 \frac{\mathrm{kW}}{\mathrm{m}^{2}}}=1.325 .891 \mathrm{~m}^{2}
$$

Os coletores solares disponíveis no mercado apresentam diferentes áreas e configurações. Adota-se, neste trabalho, coletores com área de abertura equivalente a $6 \mathrm{~m}^{2}$, característico de diversas instalações em operação. O campo solar é composto por 470 coletores solares em série e 470 coletores solares em paralelo $\left(470 \times 470 \times 6 \mathrm{~m}^{2}=1.325 .891 \mathrm{~m}^{2}\right)$.

\subsection{Ciclo de Rankine Acoplado ao Campo Solar e ao Armazenamento Térmico}

O objetivo do armazenamento térmico é, como o próprio nome sugere, armazenar energia para sua posterior utilização em um período em que não há radiação suficiente para suprir a demanda energética do Ciclo de Rankine. Neste caso, tal energia é captada pelo campo solar quando há radiação solar disponível, ou seja, durante o período diurno, armazenada e, posteriormente, destinada à geração de potência, normalmente, no período noturno. Assim, espera-se que haja geração contínua de potência, tanto durante o dia quanto durante a noite. Uma representação genérica do sistema completo: campo solar, armazenamento térmico e Ciclo de Rankine, acoplados, é apresentada na Figura 3.

O esquema da proposta implementada no TRNSYS é apresentado na Figura 4. O Armazenamento Térmico, assim como descrito no esquema, é um componente do TRNSYS (Type 430) que simula o armazenamento térmico em concreto para fluidos monofásicos (óleo HTF, água, ar). Consiste em tubos iguais, paralelos em concreto com HTF que flui através de duas direções possíveis: fluxo para baixo (normalmente o fluxo de carga entra quente) e fluxo para cima (normalmente o fluxo de descarga entra frio).
O componente type 430 apresenta as seguintes variáveis de entrada: Temperatura e taxa de fluxo entrando no armazenamento na parte superior ou inferior; Temperatura ambiente.

Os dados de saída disponibilizados por este componente são: Temperatura e taxa de fluxo deixando o armazenamento na parte superior ou inferior; Temperatura do leito nas partes superior, média e inferior; Taxas de calor efetivas no armazenamento; Energia armazenada no sistema.

Por sua vez, o componente Type 430 apresenta os seguintes parâmetros, que necessitam ser informados para a efetiva simulação: Dimensões do sistema de armazenamento; Propriedades físicas do fluido HTF; Propriedades do material de armazenamento; Coeficiente global de perda de calor do sistema; Vazão mássica do fluido HTF.

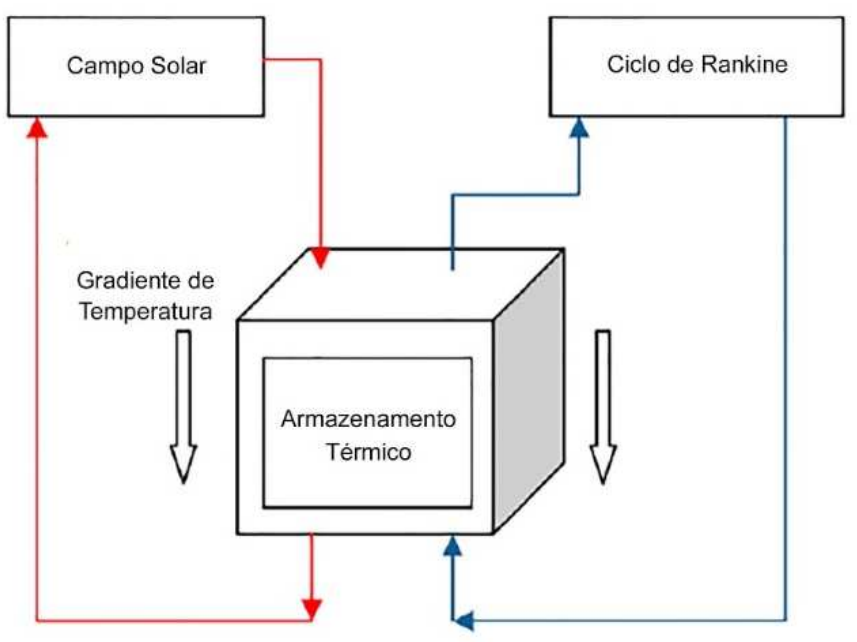

Figura 3 - Representação genérica do armazenamento térmico acoplado ao campo solar e ao Ciclo de Rankine. Adaptado de Strigari (2009)

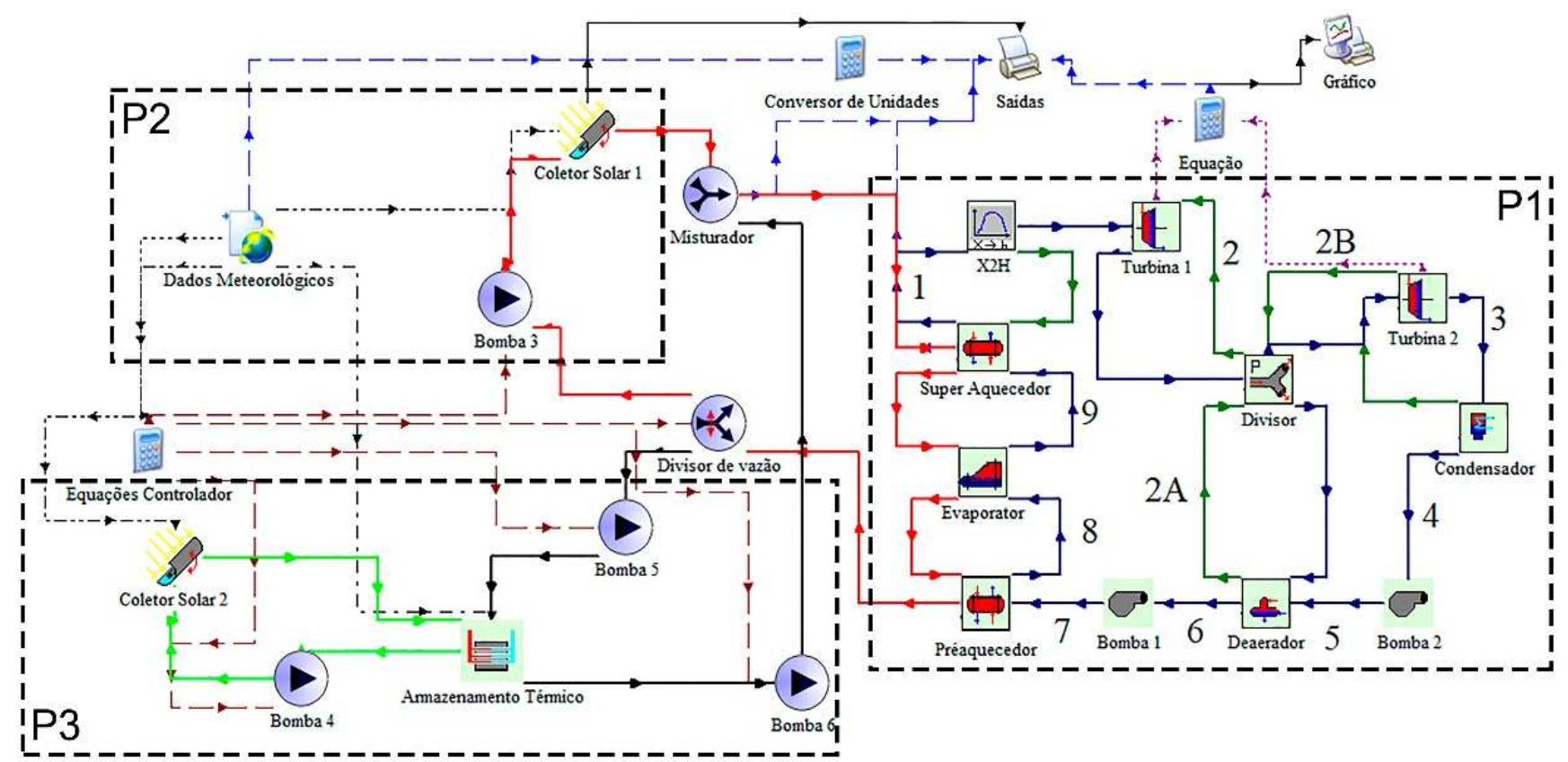

Figura 4 - Esquema do armazenamento térmico acoplado ao campo solar e ao Ciclo de Rankine no TRNSYS. 
Dimensionamento do armazenamento térmico: O armazenamento térmico deve ser dimensionado conforme às necessidades energéticas a serem supridas. Inicialmente, a energia requerida no Ciclo de Rankine é calculada através de um balanço energético no sistema, considerando nulas as perdas energéticas através das fronteiras. Os valores de capacidade calorífica da água e do vapor foram calculados pelas correlações para líquidos e gases ideais (Smith, 2007). Os valores de entalpia utilizados foram obtidos pelas tabelas de vapor considerando água líquida e vapor d'água saturados.

$Q=m_{\text {água }} C p_{\text {água }}\left(T_{\text {sai,pré aquecedor }}-T_{\text {entra,pré aquecedor }}\right)+$ $m_{\text {água }}\left(h_{\text {vs }}-h_{\text {ls }}\right)+m_{\text {vapor }} C p_{\text {vapor }}\left(T_{\text {sai,superaquecedor }}-\right.$

$T_{\text {entra,superaquecedor }}$ )

Em que:

$$
\begin{aligned}
& \dot{m}_{\text {água }}=\dot{m}_{\text {vapor }}=123 \frac{\mathrm{kg}}{\mathrm{s}} \\
& T_{\text {sai,pré aquecedor }}=311^{\circ} \mathrm{C} \\
& T_{\text {entra,pré aquecedor }}=212,38^{\circ} \mathrm{C} \\
& T_{\text {sai,superaquecedor }}=390^{\circ} \mathrm{C} \\
& T_{\text {entra,superaquecedor }}=311^{\circ} \mathrm{C} \\
& h_{\text {vs }}=2.725,47 \frac{\mathrm{kJ}}{\mathrm{kg}} \\
& h_{\text {ls }}=1.407,87 \frac{\mathrm{kJ}}{\mathrm{kg}} \\
& C p_{\text {água }}=4,31 \frac{\mathrm{kJ}}{\mathrm{kg}{ }^{\circ} \mathrm{C}} \\
& C p_{\text {vapor }}=2,03 \frac{\mathrm{kJ}}{\mathrm{kg}^{\circ} \mathrm{C}}
\end{aligned}
$$

Substituindo os valores das equações (11) a (19) na equação (10):

$$
Q=234.072 \mathrm{~kW}
$$

Considerando que a demanda noturna seja $40 \%$ da demanda máxima (20), a energia demandada neste período será:

$$
Q_{\text {noite }}=93.629 \mathrm{~kW}
$$

Considerando que o período noturno, sem radiação, dure, em média, 13 horas e corrigindo o valor de acordo com a eficiência do Ciclo de Rankine dado pela equação (5), a energia que deve ser armazenada para suprir o sistema de geração de potência neste período será:

$$
Q_{\text {armazenado }}=93.626 \frac{\mathrm{kW}}{0.379} 13 \mathrm{~h}=11.561 .575 .730 \mathrm{~kJ}
$$

Admitindo que a diferença de temperatura no concreto não será superior a $25^{\circ} \mathrm{C}\left(390^{\circ} \mathrm{C}-365^{\circ} \mathrm{C}\right)$, a massa de concreto requerida para conter a energia demandada no período noturno é dado pela equação (23), que irá variar de acordo com o material utilizado no armazenamento.

$$
m_{\text {material }}=\frac{11.561 .575 .730 \mathrm{~kJ}}{C p_{\text {material }} 25^{\circ} \mathrm{C}}
$$

\section{RESULTADOS E DISCUSSÕES}

A simulação foi realizada considerando o período de um ano (8.760 horas). Para o Ciclo de Rankine, onde todos os parâmetros se mantiveram constantes ao decorrer da simulação, a potência de $100 \mathrm{MW}$, representada pela linha vermelha na Figura 5, foi atingida constantemente. Esta é o resultado da soma global das potências geradas pelas turbinas 1 e 2 .

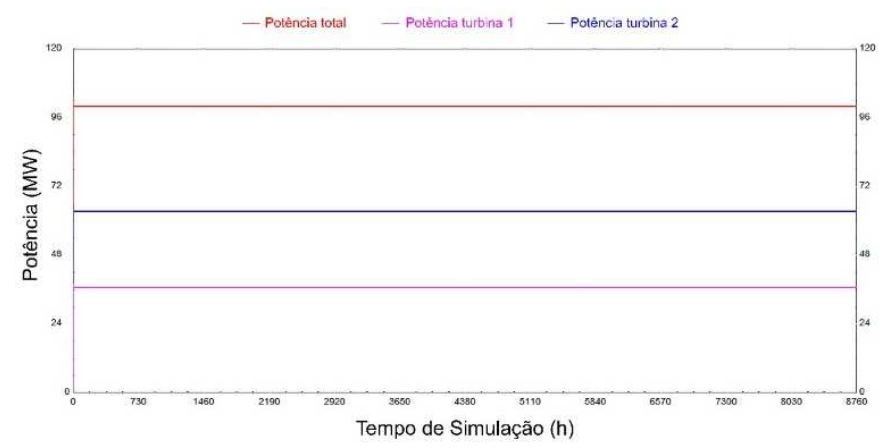

Figura 5 - Potência gerada vs tempo de simulação.

Com o campo solar acoplado ao Ciclo de Rankine, os parâmetros se tornam variáveis ao longo do tempo de simulação, devido à instabilidade e à sazonalidade da fonte de energia (Costa, 2014). Consequentemente, a potência gerada varia ao longo do tempo, como mostra a Figura 6.

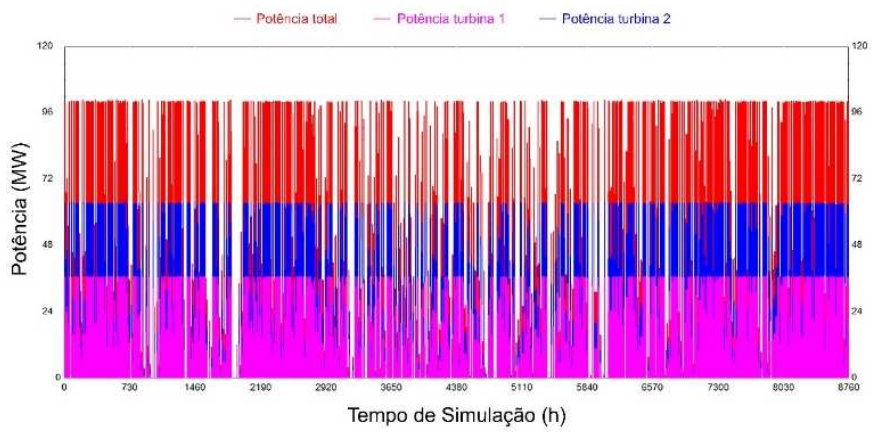

Figura 6 - Potência gerada pelo Ciclo de Rankine acoplado ao campo solar.

Na Tabela 3, é apresentada uma comparação dos parâmetros do Ciclo de Rankine, entre os valores teóricos e resultantes da simulação a fim de validar a proposta implementada no TRNSYS. Com exceção do valor da pressão na exaustão da turbina 1 , nomeada na tabela por pressão em 2 , que apresentou um erro percentual de $28 \%$, do valor da simulação em relação ao valor teórico, os demais parâmetros apresentaram valores de erro percentual inferiores a $10 \%$ Tais diferenças entre os valores teóricos e fornecidos pelo TRNSYS podem ser justificados pelas considerações adotadas durante a modelagem matemática.

Tabela 3 - Comparação entre valores teóricos e da simulação no TRNSYS para o Ciclo de Rankine.

\begin{tabular}{lcc} 
Parâmetro & $\begin{array}{c}\text { Valor } \\
\text { teórico }\end{array}$ & $\begin{array}{c}\text { Valor no } \\
\text { TRNSYS }\end{array}$ \\
\hline Entalpia $\mathrm{h}_{1} / \mathrm{kJ} / \mathrm{kg}$ & $3.065,86$ & $2.938,10$ \\
Entalpia $\mathrm{h}_{2} / \mathrm{kJ} / \mathrm{kg}$ & $2.767,37$ & $3.655,33$ \\
Entalpia $\mathrm{h}_{3} / \mathrm{kJ} / \mathrm{kg}$ & $2.019,9$ & $1.928,44$ \\
Temperatura da água pré-aquecida $/{ }^{\circ} \mathrm{C}$ & $3.10,99$ & 310,41 \\
Temperatura do vapor super. $/{ }^{\circ} \mathrm{C}$ & 390,00 & 353,54 \\
Temperatura em $4 /{ }^{\circ} \mathrm{C}$ & 28,96 & 30,00 \\
Temperatura em $7 /{ }^{\circ} \mathrm{C}$ & 212,38 & 224,33 \\
Potência total / MW & 100 & 100 \\
Pressão do Condensador / bar & 0,0400 & 0,0426 \\
Pressão em 1 / bar & 100 & 100 \\
Pressão em 2 / bar & 20 & 25,57 \\
Pressão em 7 / bar & 100 & 100 \\
Pressão em $8 /$ bar & 100 & 100 \\
Pressão em $9 /$ bar & 100 & 100 \\
\hline
\end{tabular}


A demanda energética de 100 MW foi alcançada em 804 das 8.760 horas de simulação, período correspondente a pouco mais de um mês. Por outro lado, durante 5.519 horas não houve geração de potência, período correspondente a, aproximadamente, 7 meses e meio.

Um destaque no tempo de simulação, de 1.535 horas a 1.700 horas correspondente ao período de 5 a 12 de março, permite perceber a diferença na geração de potência em dias mostrado na Figura 7, ensolarados e nublados, em que há grande variação nos valores de DNI.

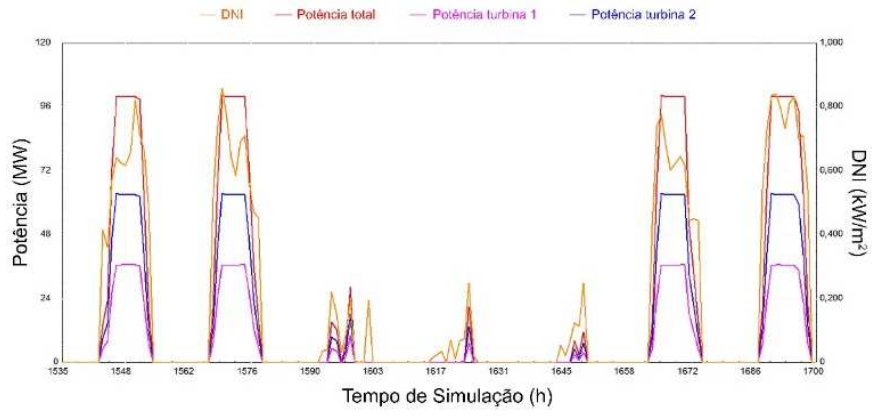

Figura 7 - Geração de potência e DNI vs tempo de simulação.

A efetividade do armazenamento térmico foi comprovada pela geração de potência ininterruptamente pela quase totalidade do ano, assim como mostra a Figura 8. A demanda de 100 MW foi obtida em 1.729 horas, o que corresponde a quase 2 meses e meio. Por 2.447 horas, equivalente a quase 3 meses e meio, a potência gerada atingiu $40 \mathrm{MW}$, valor demandado em períodos sem radiação.

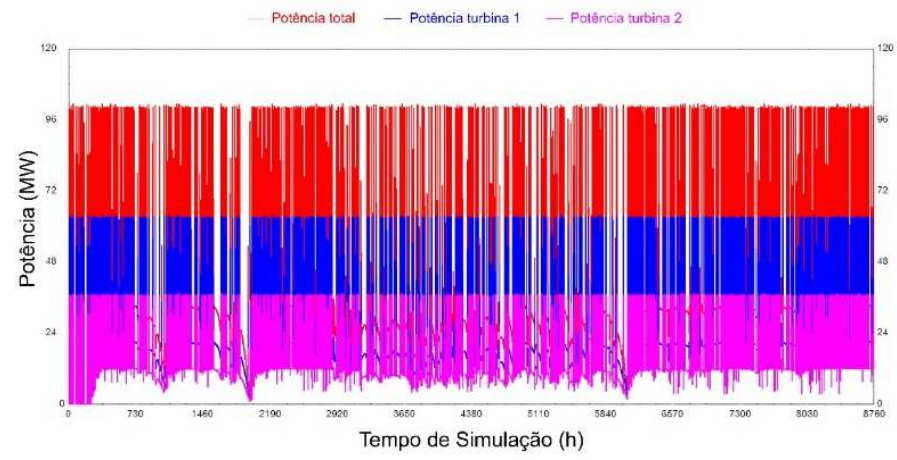

Figura 8 - Potência gerada pelo Ciclo de Rankine acoplado ao campo solar e ao armazenamento térmico.

A ausência de potência gerada foi constatada em 177 horas do ano, período equivalente a 7 dias. Além disso, o número de horas em que houve geração de potência aumentou de 3241 para 8583 com a utilização do armazenamento térmico, aumento correspondente a aproximadamente $260 \%$.

\section{CONCLUSÃO}

O desenvolvimento deste trabalho possibilitou uma avaliação de como um software pode contribuir para pesquisas e validações da implementação de projetos técnicos. Além disso, foram aplicados conceitos relacionados a áreas de termodinâmica, fenômenos de transporte, operações unitárias, projetos e balanços de massa e energia, ambos fundamentais para modelagem da proposta apresentada.

A simulação realizada constatou a efetividade da proposta do armazenamento térmico, uma vez que a utilização deste no sistema aumentou consideravelmente o número de horas em que houve geração de potência quando comparado ao sistema sem este recurso. Dessa forma, os resultados apresentados justificam a utilização de tal tecnologia para contornar a instabilidade e a sazonalidade da fonte energética, como descrito por Costa (2014).

Finalmente, destaca-se, ainda, que a seleção do material de armazenamento impacta significativamente nos custos da instalação, pois quanto maior a capacidade calorífica do material de armazenamento, menor será a massa necessária, e portanto, menores serão os custos de instalação. Neste sentido, deve-se avaliar a utilização de diferentes tipos de materiais.

Destaca-se, ainda, que o aumento ou diminuição da massa de material de armazenamento afeta o volume da instalação. O que impacta diretamente nos custos de instalação relacionados a aquisição de área para o projeto.

Dada a relevância do assunto em virtude da busca por fontes energéticas renováveis e da viabilização da implementação da tecnologia, torna-se necessário um maior desenvolvimento técnico da proposta, a partir do estudo de outros parâmetros que afetam diretamente o modelo abordado. Também, uma análise econômica voltada à instalação e operação da planta é fundamental para o conhecimento e redução dos custos a fim de tornar Plantas de Concentração Solar para geração de potência competitivas com outras fontes de energia já estabelecidas no mercado.

\section{REFER ÊNCIAS}

Corgozinho, I. M; Neto, J. H. M; Corgozinho, A. A. Modelo de simulação de uma planta solar-elétrica utilizando o software TRNSYS. V Congresso Brasileiro de Energia Solar. Recife. 2014.

Costa, S. P. L. Armazenamento de Energia Térmica Através de Materiais de Mudança de Fase. Faculdade de Engenharia. Universidade de Porto. Portugal. 2014.

Cotterman, T. "Energy storage technologies: transforming America's inteligente eletrical." 2013.

Dincer, I. Rosen, M. A. Thermal Energy Storage Systems and Applications. 2nd ed. Chichester, Reino Unido. 2011.

Durão, B. F. S. Análise dinâmica de sistemas de armazenamento sazonal de energia térmica para condicionamento ambiental de estufas. Universidade de Lisboa. Portugal. 2013.

Huggins, R. A. Energy storage. Stanford, California: Springer. 2010.

IRENA. Renewable Energy Technologies Cost Analysis Series: Concentrating Solar Power. Comprehensive Renewable Energy, v. 3, n. 2, p. 595-636, 2012.

Kuravi, S.; Trahan, J.; Goswami, D. Y.; Rahman, M. M.; Stefanakos, E. K. Thermal energy storage technologies and systems for concentrating solar power plants. Progress in Energy and Combustion Science, v. 39, p. 285-319, 2013.

McCrone, A. et. al. Global Trends in Renewable Energy Investment 2015. Frankfurt School of Finance \& Mangement, p. 16, 2015.

McGrath, M. Renewable energy surges to record levels around the world. 2016. Disponível em: <http://www.bbc.com/news/science-environment36420750>. Acessado em março de 2017. 
Moran, M. J. et. al. Fundamenntal of Engineering Thermodynamics. 8th ed. p. 466. Wiley. 2014.

Outlook, W. E. World Energy Outlook 2016. 2016.

Pantuzi, T. A. Um estudo teórico da transferência de calor em fluidos confinados em regiões cilíndricas. Guaratinguetá, São Paulo. 2006.

Pinto, C.; Catarino, J.; Correia, M.; Leite, P.; Costa, S. Energia Solar. Universidade do Porto. Portugal. 2015.

REN. Energias Renováveis 2016: Relatório da situação mundial. Ren, p. 1-31, 2016.

Sawin, J. L.; Sverrisson, F.; REN21. Renewables 2015: Global Status Report. Ren21.Net, n. September, p. 251, 2015.

Siqueira, A. M. O; Altabash, G. A; Barhouche, R; Janczewska, K; Villela, F. G; Oliveira, L. F. Simulation of parabolic trough solar power plants in Brazil. VI Congresso Brasileiro de Energia Solar. Belo Horizonte. 2016.

SolarPower Europe. Global Market Outlook for Solar Power 2015-2019. Global Market Outlook, p. 32, 2014.

Smith, J. M; Van Ness, H. C; Abbott, M. M. Introdução à Termodinâmica da Engenharia Química. Rio de Janeiro. LTC. 2007.

Strigari, D. P. Development of a concept for the autartik energy and water supply of a housing estate in an area with high average solar irradiation. Universidad Carlos III de Madrid. 2009.

Ter-Gazarian, A. G. Energy Storage for Power Systems. 2nd ed. Londres, Reino Unido. The Institution of Engineering and Technology. 2011.

Tristão, P. J. S. Encapsulação de materiais de mudança de fase (PCM) para aplicações no armazenamento de energia. Relatório Final de Projeto, Escola Superior de Tecnologia e Gestão, Instituto Politécnico de Bragança, 2014.

Wendel, Marcelo. Metodologia para simulação transiente de uma pequena central heliotérmica. Universidade Federal de Santa Catarina. Florianópolis. 2010. 